\title{
An overview of the JEM-EUSO program and results
}

\section{Bertaina ${ }^{a, *}$ on behalf of the JEM-EUSO Collaboration}

(a complete list of authors can be found at the end of the proceedings)

${ }^{a}$ Department of Physics, University of Torino \& INFN Torino, Via P. Giuria 1, Torino, Italy

E-mail: bertaina@to.infn.it

The field of UHECRs (Ultra-High energy cosmic Rays) and the understanding of particle acceleration in the cosmos, as a key ingredient to the behaviour of the most powerful sources in the universe, is of outmost importance for astroparticle physics as well as for fundamental physics and will improve our general understanding of the universe. The current main goals are to identify sources of UHECRs and their composition. For this, increased statistics is required. A space-based detector for UHECR research has the advantage of a very large exposure and a uniform coverage of the celestial sphere. The aim of the JEM-EUSO program [1] is to bring the study of UHECRs to space. The principle of observation is based on the detection of UV light emitted by isotropic fluorescence of atmospheric nitrogen excited by the Extensive Air Showers (EAS) in the Earth's atmosphere and forward-beamed Cherenkov radiation reflected from the Earth's surface or dense cloud tops. In addition to the prime objective of UHECR studies, JEMEUSO will do several secondary studies due to the instruments' unique capacity of detecting very weak UV-signals with extreme time-resolution around $1 \mu \mathrm{s}$ : meteors, Transient Luminous Events (TLE), bioluminescence, maps of human generated UV-light, searches for Strange Quark Matter (SQM) and high-energy neutrinos, and more. The JEM-EUSO program includes several missions from ground (EUSO-TA [2]), from stratospheric balloons (EUSO-Balloon [3], EUSO-SPB1 [4], EUSO-SPB2 [5]), and from space (TUS [6], Mini-EUSO [7]) employing fluorescence detectors to demonstrate the UHECR observation from space and prepare the large size missions K-EUSO [8] and POEMMA [9]. A review of the current status of the program, the key results obtained so far by the different projects, and the perspectives for the near future are presented.

$37^{\text {th }}$ International Cosmic Ray Conference (ICRC 2021)

July 12th-23rd, 2021

Online - Berlin, Germany

\footnotetext{
*Presenter
} 

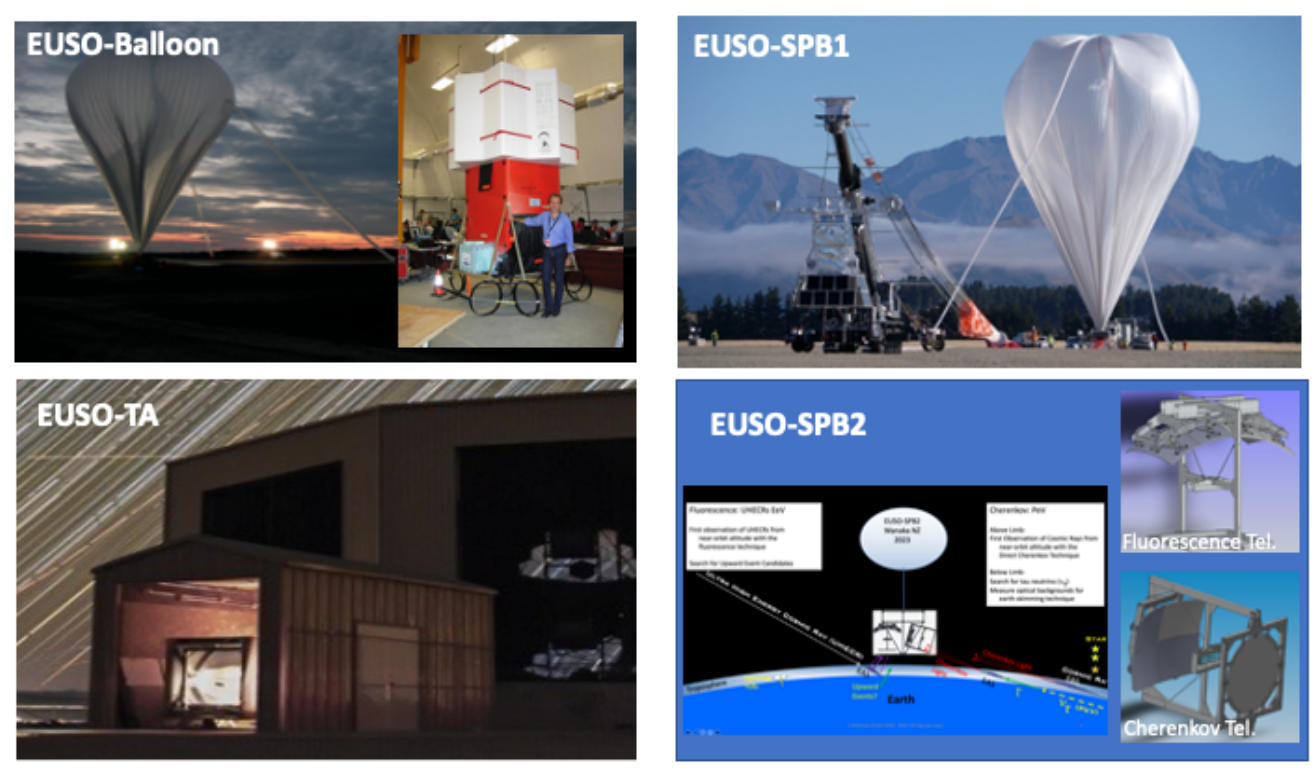

Figure 1: EUSO-TA and stratospheric balloon missions of the JEM-EUSO program. See text for details.

\section{The ground-based telescope EUSO-TA}

EUSO-TA is a ground-based telescope, installed at the TA site in Black Rock Mesa, Utah, USA (see Fig. 1). This is the first detector to successfully use a Fresnel lens based optical system and Multi-Anode Photomultipliers (MAPMT). EUSO-Balloon, EUSO-SPB1 and Mini-EUSO adopted the same observational approach. Each MAPMT has 64 channels, and they are grouped in blocks of $2 \times 2$ to form one Elementary Cell (EC). Nine ECs form one Photo-Detector Module (PDM) which has, therefore, 2304 channels encompassing a $10.6^{\circ} \times 10.6^{\circ}$ field of view (FoV) for the detection of UHECRs. The telescope is located in front of one of the fluorescence detectors of the TA experiment. Since its first operation in 2015, a few campaigns of joint observations allowed EUSO-TA to detect 9 UHECR events in 120 hours of data taking, as well as a few meteors. The limiting magnitude of 5.5 on summed frames $(\sim 3 \mathrm{~ms})$ has been established. The detector is also being calibrated using stars in the FoV, as discussed in [11]. These observations provide important data to optimise the detector technology in view of the upcoming space-based missions and to determine the sensitivity of the experiment (see [10]). The future upgrades of the detector include a new acquisition system based on Zynq board and the implementation of a self-triggering system. Simulations indicate that the new configuration should be able to have a self-trigger rate at the level of $\sim 1$ UHECR per 24 hours of accumulated measurement.

\section{The balloon program: EUSO-Balloon, EUSO-SPB1 \& EUSO-SPB2}

The JEM-EUSO program includes three stratospheric balloon missions with increasing level of performance and upgraded designs (see Fig. 1). In addition to demonstrating the capabilities of the JEM-EUSO instruments to detect and reconstruct EAS from the edge of space, they also give access to direct measurement of the UV nightglow emission and artificial UV contributions above 
ground and oceans, which are important information to optimise the design of the space-based missions. Two balloon flights have been performed so far: EUSO-Balloon (Canada, 1 night) and EUSO-SPB1 (New Zealand, 12 nights). A third one (EUSO-SPB2) is scheduled to fly in 2023.

EUSO-Balloon [3] was launched by CNES from the Timmins base in Ontario (Canada) on the moonless night of August 25, 2014. The telescope configuration was very similar to EUSO-TA and the subsequent EUSO-SPB1. After reaching the floating altitude of $\sim 38 \mathrm{~km}$, EUSO-Balloon imaged the UV intensity with a spatial and temporal resolutions of $130 \mathrm{~m}$ and $2.5 \mu \mathrm{s}$, respectively, in the wavelength range $290-430 \mathrm{~nm}$ for more than 5 hours before descending to ground. The full FoV in nadir mode was $\sim 11^{\circ}$. During 2.5 hours of EUSO-Balloon flight, a helicopter circled under the balloon operating UV flashers and a UV laser to simulate the optical signals from UHECRs, to calibrate the apparatus, and to characterise the optical atmospheric conditions. Data collected by EUSO-Balloon have been analysed to infer different information among which the response of the detector to the UV flasher and laser events, and the UV radiance from the Earth atmosphere and ground in different conditions: clear and cloudy atmosphere, forests, lakes, as well as city lights. This is relevant for a JEM-EUSO-like mission as it is one of the key parameters to estimate the exposure curve as a function of energy [12]. The results of this analysis are reported in [13] and are in the band of previous measurements confirming a good understanding of the detector performance also in this respect. The helicopter events proved to be extremely useful to understand the system's performance and to test the capability of EUSO-Balloon to detect and reconstruct signals similar to EAS [14]. The data collected by EUSO-Balloon were used together with those collected by EUSO-TA to define an internal trigger logic [15] that was implemented on the EUSO-SPB1 flight.

EUSO-SPB1 [4] was launched on April 25, 2017 from Wanaka, New Zealand, as a mission of opportunity on a NASA SPB test flight planned to circle the southern hemisphere. The telescope was an upgraded version of that used in the EUSO-Balloon mission. An autonomous internal trigger was implemented according to [15] to detect UHECRs. Prior to flight, in October 2016, the fully assembled EUSO-SPB1 detector was tested for a week at the EUSO-TA site to measure its response and to calibrate it by means of a portable Ground Laser System (GLS). Observations of Central Laser Facility (CLF), stars, meteors were performed. The $\sim 50 \%$ trigger efficiency was reached at laser energies whose luminosity is equivalent to $\sim 45^{\circ}$ inclined EAS of $\mathrm{E} \sim 3 \times 10^{18} \mathrm{eV}$ seen from above by a balloon flying at $33 \mathrm{~km}$ altitude. Unfortunately, although the instrument was showing nominal behaviour and performances, the flight was terminated prematurely in the Pacific Ocean about $300 \mathrm{~km} \mathrm{SE}$ of Easter Island after only 12 days aloft, due to a leak in the carrying balloon. During flight, $\sim 30$ hours of data were collected, the trigger rate was tipycally a few $\mathrm{Hz}$, which is compliant with JEM-EUSO requirements [16]. A deep analysis of the collected data was performed. Tracks of CRs directly crossing the detector were recognized. However, no EAS track has been clearly identified $[17,18]$. Simulations post-flight indicate that the number of expected events is $0.5-1$ in the available data sample including the role of clouds confirming pre-flight expectations for such a flight duration [19, 20].

The subsequent step of the JEM-EUSO program development is currently under realization: EUSO-SPB2 [21]. It will be equipped with 2 telescopes. One telescope will be devoted to UHECR measurements using the fluorescence technique. The Focal Surface (FS) [22] will be equipped with 3 PDMs to increase the UHECR collection power. EUSO-SPB2 will adopt a Schmidt camera with a shorter temporal resolution of $1 \mu$ s and a more efficient trigger logic to improve the sensitivity of 


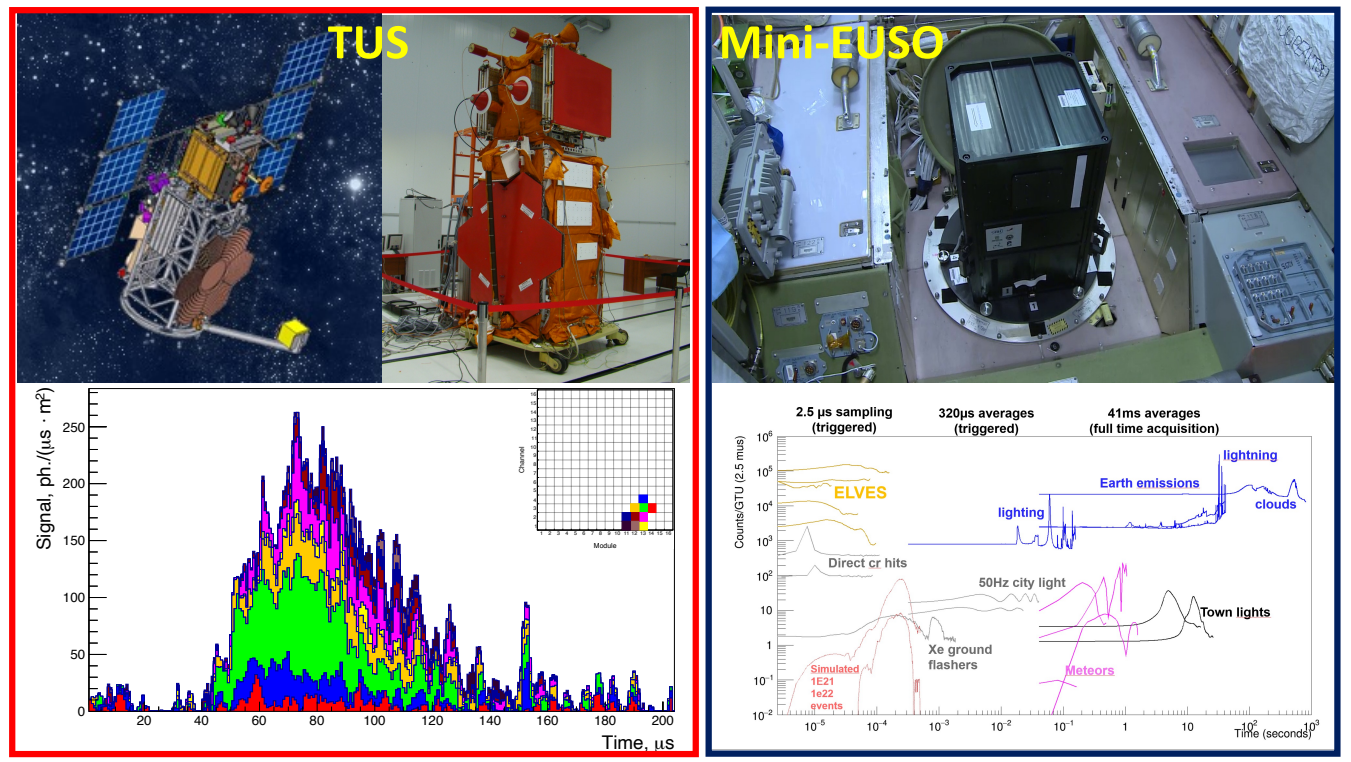

Figure 2: TUS and Mini-EUSO telescopes with examples of their observations (see text for details).

the instrument. Simulations indicate that the energy threshold is around $2 \times 10^{18} \mathrm{eV}$ and an expected detection rate of 0.6 events per night of acquisition [23]. The FS of the second telescope is based on SiPMT sensors and a dedicated electronics to detect the Cherenkov emission in air by UHECRgenerated EASs [24]. In perspective they will test the capability to detect EAS generated by $v_{\tau}$ interacting in the Earth crust [25]. For this observation the detector will be pointing slightly below the limb. The observation above the limb will allow to study UHECRs through their Cherenkov emission. EUSO-SPB2 is expected to fly in 2023 from Wanaka on a NASA Super Pressure Balloon.

\section{The first space-based missions of the program: TUS and Mini-EUSO}

The Track Ultraviolet Setup (TUS) [6] detector (see Fig. 2) was launched on April 28, 2016 as a part of the scientific payload of the Lomonosov satellite. The instrument was actively recording data till November 2017. Different scientific modes were tested: cosmic ray, lightning and meteor modes. The satellite has a sun-synchronous orbit with an inclination of $97.3^{\circ}$, a period of $\sim 94$ min, and a height of $470-500 \mathrm{~km}$. The telescope consists of two main parts: a modular Fresnel mirror-concentrator with an area of $\sim 2 \mathrm{~m}^{2}$ and 256 PMTs arranged in a $16 \times 16$ photo-receiver matrix located in the focal plane of the mirror. The pixel's FoV is $10 \mathrm{mrad}$, which corresponds to a spatial spot of $\sim 5 \mathrm{~km} \times 5 \mathrm{~km}$ at sea level. Thus, the full area observed by TUS at any moment is $\sim 80 \mathrm{~km} \times$ $80 \mathrm{~km}$. TUS is sensitive to the near UV band and in cosmic ray mode has a time resolution of 0.8 $\mu \mathrm{s}$ in a full temporal interval of 256 time steps. TUS data offer the opportunity to develop strategies in the analysis and reconstruction of events which will be essential for future space-based missions. A detailed analysis of TUS exposure and sensitivity to UHECR is presented in [26]. During its operation TUS has detected about $8 \times 10^{4}$ events that have been subject to an offline analysis to select among them those satisfying basic temporal and spatial criteria of UHECRs. A few events passed this first screening. One specific event (see Fig. 2) registered in perfect observational conditions was 
deeply scrutinized. Its phenomenology and the possible interpretations are reported in detail in [27]. Similar searches performed with Mini-EUSO support the interpretation of an anthropogenic origin of this event. However, the important result of this observation is that a space-based observatory can detect light pulses with temporal and spatial characteristics of EAS.

While TUS was conceived mainly to prove the observation of UHECRs from space with a FS-instrumentation similar to ground-based detectors, Mini-EUSO has been developed in order to test the same FS-instrumentation foreseen for K-EUSO and POEMMA. Mini-EUSO [28] is a UV telescope launched in August 2019 and installed periodically inside the ISS since October 2019, looking down the Earth from a nadir-facing window in the Russian Zvezda module. So far more than 40 sessions of about 12 hours of data taking have been performed [29]. Mini-EUSO maps the Earth in the UV range (290 - $430 \mathrm{~nm}$ ) with a spatial resolutions of $\sim 6 \mathrm{~km}$ (similar to TUS) and three different temporal resolutions of $2.5 \mu \mathrm{s}, 320 \mu \mathrm{s}$, and $41 \mathrm{~ms}$, respectively. While the $41 \mathrm{~ms}$ time range allows a continuous video-taking, the other two modes allow acquisitions of 4 packets of 128 GTUs each every 5.24s to catch fast luminous transients (flashes, lightnings, etc..). The optical system consists of 2 Fresnel lenses of $25 \mathrm{~cm}$ diameter each with a large FoV of $\sim 44^{\circ}$. Mini-EUSO energy threhsold for UHECRs is well above $10^{21} \mathrm{eV}$ [30]. Data carried down to Earth from the ISS allowed to perform the first analyses showing that Mini-EUSO observes different Earth emissions depending on the surface visible, e.g., ground, sea, or clouds as well as slow transients such as meteors (thousands of events have been identified in the data with absolute magnitude lower than +5 [31]). At shorter times scales, several hundreds of lightings (among them 17 elves [32]) have been detected and at much shorter time scales, many anthropogenic flashes presumably related to airport lights or other flashing tower lights have been acquired. Thanks to the Mini-EUSO capability to record consecutive events, this class of events is clearly identified as they repeat themselves periodically [33] and their location can be easily recognized thanks to the continuous data taking in slow mode (41 ms time frames). Some of them have characteristics similar to the TUS event previously described. So far, only one event without repetition and above ocean has been detected. However, a UHECR origin of this event has been excluded based on the light profile associated to the duration and image on the FS. Preliminary analysis of the UV maps indicate that Mini-EUSO often detects the presence of clouds [34] and that the UV light intensity recorded by Mini-EUSO is within the range of values considered in the estimation of JEM-EUSO exposure [35].

\section{The large space-based missions: K-EUSO and POEMMA}

The central objective of K-EUSO [8] is the first consistent measurement of the UHECR flux over the entire sky with almost uniform exposure. K-EUSO is a result of the joint efforts to improve the performance of the Russian KLYPVE mission [36], by employing the technologies developed for the JEM-EUSO mission, such as the optical system, focal surface detectors and the readout electronics. Since its first conception as KLYPVE, K-EUSO project has passed various modifications aimed to satisfy carrier volume and deployment capabilities on the ISS. It will be the first detector with a real capability for UHECR spectrum and anisotropy study with a yearly exposure of $\sim 2 \times 10^{4} \mathrm{~km}^{2}$ yr sr and a flat full celestial sphere coverage. The adopted optical layout is a two-lens telescope of $240 \times 120 \mathrm{~cm}^{2}$ with a FoV of 40 degree. The focal surface consists of about 40 PMDs. The temporal (sampling time is $1 \mu \mathrm{s}$ ) and spatial (angular resolution per pixel 
$0.066^{\circ}$ ) evolution of UV light recorded by K-EUSO will allow the reconstruction of the EAS, with sufficient energy and arrival direction resolutions above energies of $5 \times 10^{19} \mathrm{eV}$ [37]. Attached to the Russian MRM-1 module on-board ISS, K-EUSO is planned to operate for minimum of 2 years and it can function more than 6 years if the lifetime of the ISS is extended.

The Probe Of Extreme Multi-Messenger Astrophysics (POEMMA) mission [9] is being designed to establish charged particle astronomy from space with UHECRs and cosmic neutrinos. POEMMA will monitor colossal volumes of the Earth's atmosphere to detect EASs produced by extremely energetic cosmic messengers: cosmic neutrinos above $20 \mathrm{PeV}$ and UHECRs above 20 $\mathrm{EeV}$ over the entire sky. The POEMMA design combines the concept developed for the OWL mission [38] and the experience of the JEM-EUSO fluorescence detection camera. POEMMA is composed of two identical satellites flying in formation at $525 \mathrm{~km}$ altitude with the ability to observe overlapping regions during moonless nights at angles ranging from Nadir to just above the limb of the Earth, but also with independent pointing strategies to exploit at maximum the scientific program of the mission. Each telescope is composed of a wide $\left(45^{\circ}\right)$ ) FoV Schmidt optical system with an optical collecting area of over $6 \mathrm{~m}^{2}$. The POEMMA FS is composed of a hybrid of two types of cameras: about $90 \%$ of the FS is dedicated to the POEMMA fluorescence camera (PFC), while POEMMA Cherenkov camera (PCC) occupies the crescent moon shaped edge of the FS which images the limb of the Earth. The PFC is composed of JEM-EUSO PDMs based on MAPMTs. The typical time between images for the PFC is about $1 \mu \mathrm{sec}$. The much faster POEMMA Cherenkov camera (PCC) is composed of Silicon photo-multipliers (SiPMs) which will be tested with EUSOSPB2. The PFC registers UHECR tracks from Nadir to just below the Earth's limb (above $20 \mathrm{EeV}$ ), while the PCC registers light within the Cherenkov emission cone of up-going showers around the limb of the Earth and also from high energy cosmic rays above the limb of the Earth (above 20 $\mathrm{PeV}$ ). Simulations indicate that POEMMA has excellent performance in UHECR energy, $X_{\text {max }}$, and angular reconstruction capabilities [39], as well as high sensitivity in Target of Opportunity observations exploiting upward-moving EASs induced by Earth-interacting tau neutrinos [40]. The roadmap to the POEMMA mission is summarized in [41].

\section{Acknowledgments}

This work was partially supported by Basic Science Interdisciplinary Research Projects of RIKEN and JSPS KAKENHI Grant (22340063, 23340081, and 24244042), by the Italian Ministry of Foreign Affairs and International Cooperation, by the Italian Space Agency through the ASI INFN agreements n. 2017-8-H.0 and n. 2021-8-HH.0, by NASA award 11-APRA-0058, 16-APROBES16-0023, 17-APRA17-0066, NNX17AJ82G, NNX13AH54G, 80NSSC18K0246, 80NSSC18K0473, 80NSSC19K0626, and 80NSSC18K0464 in the USA, by the French space agency CNES, by the Deutsches Zentrum für Luft- und Raumfahrt, the Helmholtz Alliance for Astroparticle Physics funded by the Initiative and Networking Fund of the Helmholtz Association (Germany), by Slovak Academy of Sciences MVTS JEM-EUSO, by National Science Centre in Poland grants 2017/27/B/ST9/02162 and 2020/37/B/ST9/01821, by Deutsche Forschungsgemeinschaft (DFG, German Research Foundation) under GermanyÕs Excellence Strategy - EXC-2094390783311, by Mexican funding agencies PAPIIT-UNAM, CONACyT and the Mexican Space Agency (AEM), as well as VEGA grant agency project 2/0132/17, and by by State Space Cor- 
poration ROSCOSMOS and the Interdisciplinary Scientific and Educational School of Moscow University "Fundamental and Applied Space Research".

\section{References}

[1] M. Ricci (JEM-EUSO Coll.), J. Phys. Conf. Ser. 718 (2016) no.5, 052034.

[2] G. Abdellaoui et al (JEM-EUSO Coll.), Astroparticle Physics 102 (2018) 98.

[3] J.H. Adams et al (JEM-EUSO Coll.), Experimental Astronomy 40 (2015) 281.

[4] L. Wiencke and A. Olinto for the JEM-EUSO Coll., PoS(ICRC2017), 1097.

[5] J.H. Adams et al, ArXiv e-prints [arXiv]1703.04513 (2017).

[6] P. Klimov et al (TUS Coll.), Space Science Reviews 8 (2017) 1.

[7] S. Bacholle et al, ApJS, 253 (2021) 36.

[8] M. Casolino et al, PTEP 2017/12 (2017) 12A107.

[9] A. Olinto et al (POEMMA Coll.), JCAP 06 (2021) 007.

[10] F. Bisconti et al (JEM-EUSO Coll.), EUSO-TA ground based fluorescence detector: analysis of the detected events, PoS(ICRC2019) 197.

[11] Z. Plebaniak et al (JEM-EUSO Coll.), Calibration of EUSO-TA detector with stars, PoS(ICRC2019) 393.

[12] J.H. Adams et al (JEM-EUSO Coll.), Astroparticle Physics 44 (2013) 76.

[13] G. Abdellaoui et al (JEM-EUSO Coll.), Astroparticle Physics 111 (2019) 54.

[14] G. Abdellaoui et al (JEM-EUSO Coll.), J. of Instrumentation 13 (2018) 05023.

[15] G. Abdellaoui et al (JEM-EUSO Coll.), Nucl. Instr. \& Meth. A 866 (2017) 150.

[16] M. Battisti et al (JEM-EUSO Coll.), Nucl. Instr. \& Meth. A 936349 (2019).

[17] A. Diaz et al (JEM-EUSO Coll.), EUSO-SPB1: Flight data classification and Air shower search results, PoS(ICRC2019) 240.

[18] M. Vrabel et al (JEM-EUSO Coll.), Machine Learning Approach for Air Shower Recognition in the EUSO-SPB Experiment Data, PoS(ICRC2019) 456.

[19] J. Eser et al (JEM-EUSO Coll.), EUSO-SPB1 overview, PoS(ICRC2019) 247.

[20] K. Shinozaki et al (JEM-EUSO Coll.), An estimation of the exposure of air shower detectionby the EUSO-SPB1 mission, PoS(ICRC2019) 427.

[21] J. Eser et al (JEM-EUSO Coll.), EUSO-SPB2 science \& mission status, PoS(ICRC2021) 235. 
[22] G. Osteria et al (JEM-EUSO Coll.), The Fluorescence Telescope on board EUSO-SPB2 for the detection of Ultra High Energy Cosmic Rays, PoS(ICRC2021) 403.

[23] G. Filippatos et al (JEM-EUSO Coll.), Expected Performance of the EUSO-SPB2 Fluorescence Telescope, PoS(ICRC2021) 330.

[24] M. Bagheri et al (JEM-EUSO Coll.), Overview of Cherenkov Telescope onboard EUSO-SPB2 for the Detection of Ultra-High Energy Neutrinos, PoS(ICRC2021) 1091.

[25] A. Cummings et al, [ArXiv]2105.03255 (2021).

[26] F. Fenu et al (TUS Coll.), Estimation of the exposure of the TUS space based cosmic ray observatory, PoS(ICRC2021) 752.

[27] B.A. Khrenov et al. (TUS Coll.), JCAP 03 (2020) 033.

[28] M. Casolino et al (JEM-EUSO Coll.), First results from the Mini-EUSO telescope on board the International Space Station, PoS(ICRC2021) 886.

[29] G. Cambiè et al (JEM-EUSO Coll.), Integration and qualification of the Mini-EUSO telescope on board the ISS, PoS(ICRC2021) 1001.

[30] F. Fenu et al (JEM-EUSO Coll.), Simulation studies for the Mini-EUSO detector, PoS(ICRC2021) 757.

[31] L. Piotrowski et al (JEM-EUSO Coll.), Towards observations of nuclearites with Mini-EUSO, PoS(ICRC2021) 1181.

[32] L. Marcelli et al (JEM-EUSO Coll.), Observations of Transient Luminous Events with the Mini-EUSO telescope on board the ISS, PoS(ICRC2021) 971.

[33] M. Battisti et al (JEM-EUSO Coll.), Overview of the Mini-EUSO $\mu$ s trigger logic performance, PoS(ICRC2021) 411.

[34] A. Golzio et al (JEM-EUSO Coll.), A study on UV emission from clouds with Mini-EUSO, PoS(ICRC2021) 417.

[35] K. Shinozaki et al (JEM-EUSO Coll.), Measurement of UV light emission of the nighttime Earth by Mini-EUSO for spaceObased UHECR observations, PoS(ICRC2021) 1165.

[36] B. Khrenov et al., Nuclear Physics B Proceedings Supplements, 113/1 (2002) 115.

[37] F. Fenu et al (JEM-EUSO Coll.), A performance study of the K-EUSO space based observatory, PoS(ICRC2021) 754.

[38] F.W. Stecker et al, Nucl. Phys. B 136C (2004) 433.

[39] L. Anchordoqui et al., Phys. Rev. D 101 (2020) 023012.

[40] T. Venters et al., Phys. Rev. D 102 (2020) 123013.

[41] A. Olinto et al (POEMMA Coll.), Roadmap to the POEMMA mission, PoS(ICRC2021) 754. 


\section{Full Authors List: JEM-EUSO Collaboration}

G. Abdellaoui ${ }^{a h}$, S. Abe ${ }^{f q}$, J.H. Adams Jr. ${ }^{p d}$, D. Allard ${ }^{c b}$, G. Alonso $^{m d}$, L. Anchordoqui ${ }^{p e}$, A. Anzalone $e^{e h, e d}$, E. Arnone $e^{e k, e l}$, K. Asano ${ }^{f e}$, R. Attallah ${ }^{a c}$, H. Attouiaa, M. Ave Pernas ${ }^{m c}$, M. Bagheri ${ }^{p h}$, J. Baláz $z^{l a}$, M. Bakiri ${ }^{a a}$, D. Barghini ${ }^{e l, e k}$, S. Bartocci ${ }^{e i, e j}$, M. Battisti ${ }^{e k, e l}$, J. Bayer ${ }^{d d}$, B. Beldjilali ${ }^{a h}$, T. Belenguer ${ }^{m b}$, N. Belkhalfa ${ }^{a a}$, R. Bellotti ${ }^{e a, e b}$, A.A. Belov ${ }^{k b}$, K. Benmessai ${ }^{a a}$, M. Bertaina ${ }^{e k, e l}$, P.F. Bertone ${ }^{p f}$, P.L. Biermann ${ }^{d b}$, F. Bisconti ${ }^{e l, e k}$, C. Blaksley ${ }^{f t}$, N. Blanc ${ }^{o a}$, S. BlinBondil $^{c a, c b}$, P. Bobik ${ }^{l a}$, M. Bogomilov $b a$, K. Bolmgren ${ }^{n a}$, E. Bozzo ${ }^{o b}$, S. Briz ${ }^{p b}$, A. Bruno ${ }^{e h, e d}$, K.S. Caballero ${ }^{h d}$, F. Cafagna ${ }^{e a}$, G. Cambiéei,ej, D. Campana ${ }^{e f}$, J-N. Capdevielle ${ }^{c b}$, F. Capel ${ }^{d e}$,

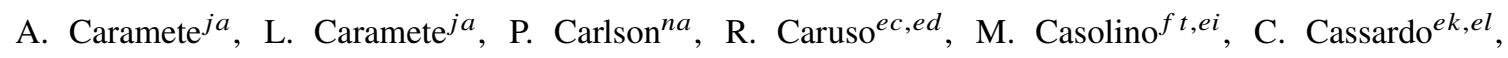

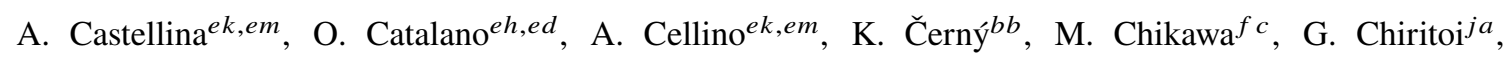

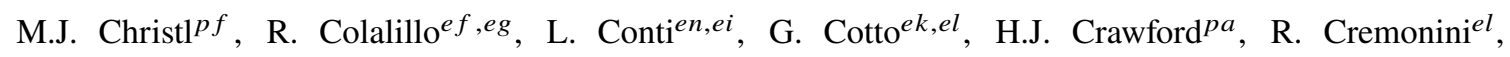
A. Creusot ${ }^{c b}$, A. de Castro Gónzalez ${ }^{p b}$, C. de la Taille ${ }^{c a}$, L. del Peral ${ }^{m c}$, A. Diaz Damian ${ }^{c c}$, R. Diesing ${ }^{p b}$, P. Dinaucourt ${ }^{c a}$, A. Djakonow ${ }^{i a}$, T. Djemil ${ }^{a c}$, A. Ebersoldt ${ }^{d b}$, T. Ebisuzaki ${ }^{f t}$, J. Eser ${ }^{p b}$, F. Fenu ${ }^{e k, e l}$, S. Fernández-González ${ }^{m a}$, S. Ferrarese ${ }^{e k, e l}$, G. Filippatos ${ }^{p c}$, W.I. Finch ${ }^{p c}$ C. Fornaro $^{e n, e i}$, M. Fouka $^{a b}$, A. Franceschi ${ }^{e e}$, S. Franchini ${ }^{m d}$, C. Fuglesang ${ }^{n a}$, T. Fujii ${ }^{f g}$, M. Fukushima ${ }^{f e}$, P. Galeotti ${ }^{e k, e l}$, E. GarcíaOrtega $^{m a}$, D. Gardiolek,em ${ }^{e k}$ G.K. Garipov ${ }^{k b}$, E. Gascón ${ }^{m a}$, E. Gazda ${ }^{p h}$, J. Gencil ${ }^{l b}$, A. Golzio ${ }^{e k, e l}$, C. González Alvarado ${ }^{m b}$, P. Gorodetzky ${ }^{f t}$, A. Green ${ }^{p c}$, F. Guarino ${ }^{e f, e g}$, C. Guépin ${ }^{p l}$, A. Guzmán ${ }^{d d}$,

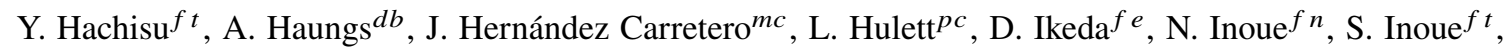

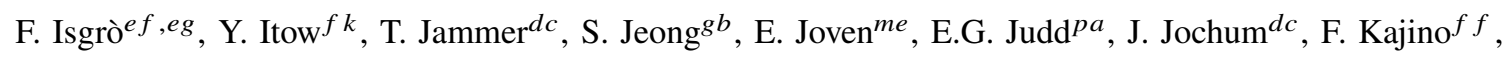
T. Kajino ${ }^{f i}$, S. Kalli ${ }^{a f}$, I. Kaneko ${ }^{f t}$, Y. Karadzhov ${ }^{b a}$, M. Kasztelan ${ }^{i a}$, K. Katahira ${ }^{f t}$, K. Kawai ${ }^{f t}$, Y. Kawasaki $^{f t}$, A. Kedadra ${ }^{a a}$, H. Khales ${ }^{a a}$, B.A. Khrenov ${ }^{k b}$, Jeong-Sook Kim ${ }^{g a}$, Soon-Wook Kim ${ }^{g a}$, M. Kleifges ${ }^{d b}$, P.A. Klimov ${ }^{k b}$, D. Kolev ${ }^{b a}$, I. Kreykenbohm ${ }^{d a}$, J.F. Krizmanic ${ }^{p f, p k}$, K. Królik ${ }^{i a}$, V. Kungel ${ }^{p c}$, Y. Kurihara ${ }^{f s}$, A. Kusenko ${ }^{f r}$,pe, E. Kuznetsov ${ }^{p d}$, H. Lahmar ${ }^{a}$, F. Lakhdari ${ }^{a g}$, J. Licandro ${ }^{m e}$, L. López Campano ${ }^{m a}$, F. López Martínez ${ }^{p b}$, S. Mackovjak ${ }^{l a}$, M. Mahdi ${ }^{a a}$, D. Mandát ${ }^{b c}$, M. Manfrin ${ }^{e k, e l}$, L. Marcelli ${ }^{e i}$, J.L. Marcos ${ }^{m a}$, W. Marszał ${ }^{i a}$, Y. Martín ${ }^{m e}$, O. Martinez ${ }^{h c}$, K. Mase ${ }^{f a}$, R. Matev ${ }^{b a}$, J.N. Matthews ${ }^{p g}$, N. Mebarki ${ }^{a d}$, G. Medina-Tanco ${ }^{h a}$, A. Menshikov ${ }^{d b}$, A. Merino ${ }^{m a}$, M. Mese $^{e f, e g}$, J. Meseguer ${ }^{m d}$, S.S. Meyer ${ }^{p b}$, J. Mimouni ${ }^{a d}$, H. Miyamoto ${ }^{e k, e l}$, Y. Mizumoto ${ }^{f i}$, A. Monaco ${ }^{e a, e b}$,

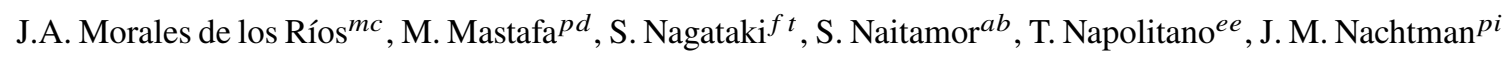

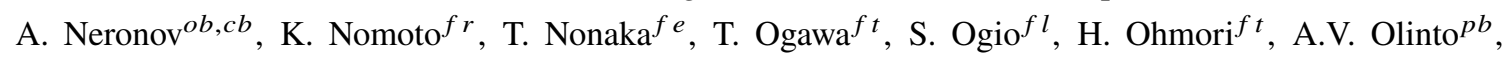
Y. Onel ${ }^{p i}$ G. Osteria ${ }^{e f}$, A.N. Otte ${ }^{p h}$, A. Pagliaro ${ }^{e h, e d}$, W. Painter ${ }^{d b}$, M.I. Panasyuk ${ }^{k b}$, B. Panico ${ }^{e f}$, E. Parizot ${ }^{c b}$, I.H. Park ${ }^{g b}$, B. Pastircak ${ }^{l a}$, T. Paul ${ }^{p e}$, M. Pech ${ }^{b b}$, I. Pérez-Grande ${ }^{m d}$, F. Perfetto ${ }^{e f}$, T. Peter $^{o c}$, P. Picozza ${ }^{e i, e j, f t}$, S. Pindado ${ }^{m d}$, L.W. Piotrowski ${ }^{i b}$, S. Piraino ${ }^{d d}$, Z. Plebaniak ${ }^{e k, e l, i a}$, A. Pollini ${ }^{o a}$, E.M. Popescu ${ }^{j a}$, R. Prevete ${ }^{e f}, e g$, G. Prévôt ${ }^{c b}$, H. Prieto ${ }^{m c}$, M. Przybylak ${ }^{i a}$, G. Puehlhofer ${ }^{d d}$, M. Putis ${ }^{l a}$, P. Reardon ${ }^{p d}$, M.H.. Reno ${ }^{p i}$, M. Reyes ${ }^{m e}$, M. Ricciee, M.D. Rodríguez Frías ${ }^{m c}$, O.F. Romero Matamala ${ }^{p h}$, F. Ronga ${ }^{e e}$, M.D. Sabau ${ }^{m b}$, G. Saccáec,ed , G. Sáez Cano ${ }^{m c}$, H. Sagawa ${ }^{f e}$, Z. Sahnoune ${ }^{a b}$, A. Saito ${ }^{f g}$, N. Sakaki ${ }^{f t}$, H. Salazar ${ }^{h c}$, J.C. Sanchez Balanzar ${ }^{h a}$, J.L. Sánchez ${ }^{m a}$, A. Santangelo ${ }^{d d}$, A. SanzAndrés $^{m d}$, M. Sanz Palomino ${ }^{m b}$, O.A. Saprykin ${ }^{k c}$, F. Sarazin ${ }^{p c}$, M. Sato ${ }^{f o}$, A. Scagliola ${ }^{e a, e b}$, T. Schanz ${ }^{d d}$, H. Schieler ${ }^{d b}$, P. Schovánek ${ }^{b c}$, V. Scotti ${ }^{e f, e g}$, M. Serra ${ }^{m e}$, S.A. Sharakin ${ }^{k b}$, H.M. Shimizu ${ }^{f j}$, K. Shinozaki ${ }^{i a}$, J.F. Soriano ${ }^{p e}$, A. Sotgiu ${ }^{e i, e j}$, I. Stan ${ }^{j a}$, I. Strharskýla, N. Sugiyama ${ }^{f j}$, D. Supanitsky ${ }^{h a}$,

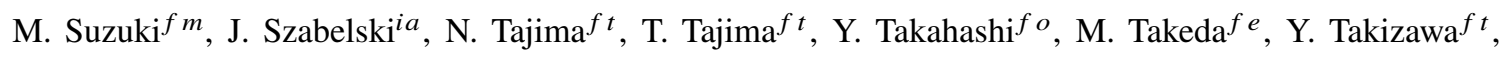
M.C. Talai ${ }^{a c}$, Y. Tameda ${ }^{f p}$, C. Tenzer ${ }^{d d}$, S.B. Thomas ${ }^{p g}$, O. Tibolla ${ }^{h e}$, L.G. Tkachev ${ }^{k a}$, T. Tomida ${ }^{f h}$,

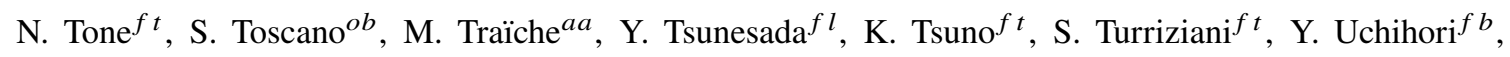


O. Vaduvescu ${ }^{m e}$, J.F. Valdés-Galicia ${ }^{h a}$, P. Vallania ${ }^{e k, e m}$, L. Valore ${ }^{e f, e g}$, G. Vankova-Kirilova ${ }^{b a}$, T. M. Venters ${ }^{p j}$, C. Vigorito ${ }^{e k, e l}$, L. Villaseñor ${ }^{h b}$, B. Vlcek $^{m c}$, P. von Ballmoos $^{c c}$, M. Vrabel $^{l b}$, S. Wada $^{f t}$, J. Watanabe ${ }^{f i}$, J. Watts Jr. ${ }^{p d}$, R. Weigand Muñoz ${ }^{m a}$, A. Weindl ${ }^{d b}$, L. Wiencke ${ }^{p c}$, M. Wille ${ }^{d a}$, J. Wilms ${ }^{d a}$,

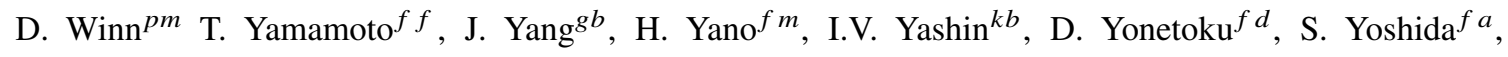
R. Young ${ }^{p f}$, I.S Zgura ${ }^{j a}$, M.Yu. Zotov ${ }^{k b}$, A. Zuccaro Marchi ${ }^{f t}$

${ }^{a}$ Centre for Development of Advanced Technologies (CDTA), Algiers, Algeria

${ }^{a b}$ Dep. Astronomy, Centre Res. Astronomy, Astrophysics and Geophysics (CRAAG), Algiers, Algeria

${ }^{a c}$ LPR at Dept. of Physics, Faculty of Sciences, University Badji Mokhtar, Annaba, Algeria

${ }^{a d}$ Lab. of Math. and Sub-Atomic Phys. (LPMPS), Univ. Constantine I, Constantine, Algeria

af Department of Physics, Faculty of Sciences, University of M'sila, M'sila, Algeria

ag Research Unit on Optics and Photonics, UROP-CDTA, Sétif, Algeria

$a h$ Telecom Lab., Faculty of Technology, University Abou Bekr Belkaid, Tlemcen, Algeria

ba St. Kliment Ohridski University of Sofia, Bulgaria

$b b$ Joint Laboratory of Optics, Faculty of Science, Palacký University, Olomouc, Czech Republic

${ }^{b c}$ Institute of Physics of the Czech Academy of Sciences, Prague, Czech Republic

ca Omega, Ecole Polytechnique, CNRS/IN2P3, Palaiseau, France

${ }^{c b}$ Université de Paris, CNRS, AstroParticule et Cosmologie, F-75013 Paris, France

${ }^{c c}$ IRAP, Université de Toulouse, CNRS, Toulouse, France

da ECAP, University of Erlangen-Nuremberg, Germany

${ }^{d b}$ Karlsruhe Institute of Technology (KIT), Germany

$d c$ Experimental Physics Institute, Kepler Center, University of Tübingen, Germany

$d d$ Institute for Astronomy and Astrophysics, Kepler Center, University of Tübingen, Germany

de Technical University of Munich, Munich, Germany

ea Istituto Nazionale di Fisica Nucleare - Sezione di Bari, Italy

${ }^{e b}$ Universita' degli Studi di Bari Aldo Moro and INFN - Sezione di Bari, Italy

ec Dipartimento di Fisica e Astronomia "Ettore Majorana", Universita' di Catania, Italy

$e d$ Istituto Nazionale di Fisica Nucleare - Sezione di Catania, Italy

$e e$ Istituto Nazionale di Fisica Nucleare - Laboratori Nazionali di Frascati, Italy

ef Istituto Nazionale di Fisica Nucleare - Sezione di Napoli, Italy

eg Universita' di Napoli Federico II - Dipartimento di Fisica "Ettore Pancini", Italy

$e h$ INAF - Istituto di Astrofisica Spaziale e Fisica Cosmica di Palermo, Italy

${ }^{e i}$ Istituto Nazionale di Fisica Nucleare - Sezione di Roma Tor Vergata, Italy

ej Universita' di Roma Tor Vergata - Dipartimento di Fisica, Roma, Italy

${ }^{e k}$ Istituto Nazionale di Fisica Nucleare - Sezione di Torino, Italy

${ }^{e l}$ Dipartimento di Fisica, Universita' di Torino, Italy

em Osservatorio Astrofisico di Torino, Istituto Nazionale di Astrofisica, Italy

en Uninettuno University, Rome, Italy

$f$ a Chiba University, Chiba, Japan

$f b$ National Institutes for Quantum and Radiological Science and Technology (QST), Chiba, Japan

$f c$ Kindai University, Higashi-Osaka, Japan

$f d$ Kanazawa University, Kanazawa, Japan

$f e$ Institute for Cosmic Ray Research, University of Tokyo, Kashiwa, Japan 
$f f$ Konan University, Kobe, Japan

$f g$ Kyoto University, Kyoto, Japan

$f^{h}$ Shinshu University, Nagano, Japan

${ }^{f i}$ National Astronomical Observatory, Mitaka, Japan

$f j$ Nagoya University, Nagoya, Japan

$f k$ Institute for Space-Earth Environmental Research, Nagoya University, Nagoya, Japan

${ }^{f l}$ Graduate School of Science, Osaka City University, Japan

${ }^{f m}$ Institute of Space and Astronautical Science/JAXA, Sagamihara, Japan

${ }^{f n}$ Saitama University, Saitama, Japan

$f o$ Hokkaido University, Sapporo, Japan

$f p$ Osaka Electro-Communication University, Neyagawa, Japan

$f q$ Nihon University Chiyoda, Tokyo, Japan

$f r$ University of Tokyo, Tokyo, Japan

$f s$ High Energy Accelerator Research Organization (KEK), Tsukuba, Japan

${ }^{f t}$ RIKEN, Wako, Japan

${ }^{g} a$ Korea Astronomy and Space Science Institute (KASI), Daejeon, Republic of Korea

${ }^{g b}$ Sungkyunkwan University, Seoul, Republic of Korea

ha Universidad Nacional Autónoma de México (UNAM), Mexico

$h b$ Universidad Michoacana de San Nicolas de Hidalgo (UMSNH), Morelia, Mexico

$h c$ Benemérita Universidad Autónoma de Puebla (BUAP), Mexico

$h d$ Universidad Autónoma de Chiapas (UNACH), Chiapas, Mexico

he Centro Mesoamericano de Física Teórica (MCTP), Mexico

ia National Centre for Nuclear Research, Lodz, Poland

${ }^{i b}$ Faculty of Physics, University of Warsaw, Poland

${ }^{j a}$ Institute of Space Science ISS, Magurele, Romania

$k a$ Joint Institute for Nuclear Research, Dubna, Russia

$k b$ Skobeltsyn Institute of Nuclear Physics, Lomonosov Moscow State University, Russia

$k c$ Space Regatta Consortium, Korolev, Russia

la Institute of Experimental Physics, Kosice, Slovakia

$l b$ Technical University Kosice (TUKE), Kosice, Slovakia

ma Universidad de León (ULE), León, Spain

$m b$ Instituto Nacional de Técnica Aeroespacial (INTA), Madrid, Spain

$m c$ Universidad de Alcalá (UAH), Madrid, Spain

$m d$ Universidad Politécnia de madrid (UPM), Madrid, Spain

me Instituto de Astrofísica de Canarias (IAC), Tenerife, Spain

na KTH Royal Institute of Technology, Stockholm, Sweden

oa Swiss Center for Electronics and Microtechnology (CSEM), Neuchâtel, Switzerland

$o b$ ISDC Data Centre for Astrophysics, Versoix, Switzerland

${ }^{o c}$ Institute for Atmospheric and Climate Science, ETH Zürich, Switzerland

pa Space Science Laboratory, University of California, Berkeley, CA, USA

${ }^{p b}$ University of Chicago, IL, USA

$p c$ Colorado School of Mines, Golden, CO, USA

$p d$ University of Alabama in Huntsville, Huntsville, AL; USA 
$p e$ Lehman College, City University of New York (CUNY), NY, USA

$p f$ NASA Marshall Space Flight Center, Huntsville, AL, USA

$p g$ University of Utah, Salt Lake City, UT, USA

$p h$ Georgia Institute of Technology, USA

${ }^{p i}$ University of Iowa, Iowa City, IA, USA

${ }^{p j}$ NASA Goddard Space Flight Center, Greenbelt, MD, USA

${ }^{p k}$ Center for Space Science \& Technology, University of Maryland, Baltimore County, Baltimore, MD, USA

${ }^{p l}$ Department of Astronomy, University of Maryland, College Park, MD, USA

${ }^{p m}$ Fairfield University, Fairfield, CT, USA 